\title{
METHOD OF SEMIDISCRETIZATION IN TIME TO NONLINEAR RETARDED DIFFERENTIAL EQUATIONS WITH NONLOCAL HISTORY CONDITIONS
}

\section{S. AGARWAL and D. BAHUGUNA}

\author{
Received 4 January 2004
}

\begin{abstract}
This paper deals with the applications of the method of semidiscretization in time to a nonlinear retarded differential equation with a nonlocal history condition. We establish the existence and uniqueness of a strong solution. Finally, we consider some applications of the abstract results.
\end{abstract}

2000 Mathematics Subject Classification: 34K30, 34G20, 47H06.

1. Introduction. Consider the following nonlinear retarded differential equation in a real Hilbert space $H$ :

$$
\begin{gathered}
u^{\prime}(t)+A u(t)=f\left(t, u(t), u\left(r_{1}(t)\right), u\left(r_{2}(t)\right), \ldots, u\left(r_{m}(t)\right)\right), \quad t \in(0, T], \\
h\left(u_{[-\tau, 0]}\right)=\phi_{0}, \quad \text { on }[-\tau, 0],
\end{gathered}
$$

where $0<\tau, T<\infty, \phi_{0} \in \mathscr{C}_{0}:=C([-\tau, 0] ; H)$, the nonlinear operator $A$ is single valued and maximal monotone defined from the domain $D(A) \subset H$ into $H$, the nonlinear map $f$ is defined from $[0, T] \times H^{m+1}$ into $H$, the map $h$ is defined from $\mathscr{C}_{0}$ into $\mathscr{C}_{0}$, and $\psi_{[-\tau, 0]}$ is the restriction of $\psi \in \mathscr{C}_{T}:=C([-\tau, T] ; H)$ on $[-\tau, 0]$. Here $\mathscr{C}_{t}:=C([-\tau, t] ; H)$ for $t \in[0, T]$ is the Banach space of all continuous functions from $[-\tau, t]$ into $H$ endowed with the supremum norm

$$
\|\phi\|_{t}:=\sup _{-\tau \leq \eta \leq t}\|\phi(\eta)\|, \quad \phi \in \mathscr{C}_{t}
$$

where $\|\cdot\|$ represents the norm in $H$ and the functions $r_{i}:[0, T] \rightarrow[-\tau, T], i=1,2, \ldots, m$.

The existence and uniqueness results for (1.1) may also be applied to the particular case, namely, the retarded functional differential equation

$$
\begin{gathered}
u^{\prime}(t)+A u(t)=f\left(t, u(t), u\left(t-\tau_{1}\right), u\left(t-\tau_{2}\right), \ldots, u\left(t-\tau_{m}\right)\right), \quad t \in(0, T], \\
u=\phi_{0}, \quad \text { on }[-\tau, 0],
\end{gathered}
$$

where $0<\boldsymbol{\tau}_{i}<T$ and $\boldsymbol{\tau}=\max \left\{\boldsymbol{\tau}_{1}, \boldsymbol{\tau}_{2}, \ldots, \boldsymbol{\tau}_{m}\right\}$.

For the earlier works on existence, uniqueness, and stability of various types of solutions of differential and functional differential equations with nonlocal conditions, we refer to Byszewski and Lakshmikantham [7], Byszewski [6], Balachandran and Chandrasekaran [4], Lin and Liu [11], and the references cited in these papers. 
Our aim is to extend the application of the method of semidiscretization in time, also known as the method of lines, to (1.1). For the applications of the method of lines to nonlinear evolution and nonlinear functional evolution equations, we refer to Kartsatos and Parrott [9, 10], Kartsatos [8], Bahuguna and Raghavendra [3], Bahuguna [1], and the references cited in these papers.

Suppose that there is $\chi \in \mathscr{C}_{T}$ such that $h\left(\chi_{[-\tau, 0]}\right)=\phi_{0}$ on $[-\tau, 0]$ and $\chi(0) \in D(A)$. We prove the existence of a strong solution $u$ of (1.1) under the assumptions of Theorem 2.4 , stated in the next section, in the sense that there exists a unique function $u \in \mathscr{b}_{T}$ such that $u(t) \in D(A)$ for a.e. $t \in[0, T], u$ is differentiable a.e. on $[0, T]$, and

$$
\begin{gathered}
u^{\prime}(t)+A u(t)=f\left(t, u(t), u\left(r_{1}(t)\right), \ldots, u\left(r_{m}(t)\right)\right), \quad \text { a.e. } t \in[0, T], \\
u_{[-\tau, 0]}=\chi_{[-\tau, 0]}, \quad \text { on }[-\tau, 0] .
\end{gathered}
$$

Finally, we show that $u$ is unique if and only if $\chi \in \mathscr{C}_{T}$ satisfying $h\left(\chi_{[-\tau, 0]}\right)=\phi_{0}$ is unique up to $[-\tau, 0]$. We also consider some applications of the abstract results.

2. Preliminaries and main result. Let $H$ be a real Hilbert space. Let $(x, y)$ be the inner product of $x, y \in H$. We assume the following conditions.

(A1) The operator $A: D(A) \subset H \rightarrow H$ is maximal monotone, that is,

$$
(A x-A y, x-y) \geq 0, \quad \forall x, y \in D(A), \quad R(I+A)=H,
$$

where $R(\cdot)$ is the range of an operator.

(A2) The map $h: \mathscr{C}_{0} \rightarrow \mathscr{C}_{0}$ and there exists $\chi \in \mathscr{C}_{T}$ such that $h\left(\chi_{[-\tau, 0]}\right)=\phi_{0}$ and $\chi(0) \in D(A)$.

(A3) The nonlinear map $f:[0, T] \times H^{m+1} \rightarrow H$ satisfies a local Lipschitz-like condition

$$
\left\|f\left(t, u_{1}, u_{2}, \ldots, u_{m+1}\right)-f\left(s, v_{1}, v_{2}, \ldots, v_{m+1}\right)\right\| \leq L_{f}(r)\left[|t-s|+\sum_{i=1}^{m+1}\left\|u_{i}-v_{i}\right\|\right],
$$

for all $\left(u_{1}, u_{2}, \ldots, u_{m+1}\right)$ and $\left(v_{1}, v_{2}, \ldots, v_{m+1}\right)$ in $B_{r}\left(H^{m+1},(\chi(0), \ldots, \chi(0))\right)$ and $t \in[0, T]$, where $L_{f}: \mathbb{R}_{+} \rightarrow \mathbb{R}_{+}$is a nondecreasing function and, for $r>0$,

$$
B_{r}\left(H^{m+1},(\chi(0), \ldots, \chi(0))\right)=\left\{\left(u_{1}, \ldots, u_{m+1}\right) \in H^{m+1}: \sum_{i=1}^{m+1}\left\|u_{i}-\chi(0)\right\| \leq r\right\} .
$$

(A4) For $i=1,2, \ldots, m$, the maps $r_{i}:[0, T] \rightarrow[-\tau, T]$ are continuous satisfying the delay property $r_{i}(t) \leq t$ for $t \in[0, T]$.

Further, if $A$ is monotone, $1+\alpha A$ is invertible for $\alpha>0$ and the inverse operator $(1+\alpha A)^{-1}$ is Lipschitz continuous, that is,

$$
\left\|(1+\alpha A)^{-1} u-(1+\alpha A)^{-1} v\right\| \leq\|u-v\|, \quad u, v \in D\left((1+\alpha A)^{-1}\right) .
$$

Also, the inverse of an invertible monotonic operator in Hilbert space $H$ is monotonic, but it might not be true in the general case. 
For an $m$-monotonic operator $A$, we introduce the following sequences of operator $(n=1,2, \ldots)$ :

$$
\begin{gathered}
J_{n}=\left(1+n^{-1} A\right)^{-1}, \\
A_{n}=A J_{n}=n\left(I-J_{n}\right),
\end{gathered}
$$

where $A J_{n}$ denotes the composition of the two maps $A$ and $J_{n}$. The $J_{n}$ and $A_{n}$ are defined everywhere on $H$. We recall simple lemmas that we will have occasions to use. More details may be found in [5].

LemMA 2.1. Let $A$ be m-monotonic. $J_{n}$ and $A_{n}$ are uniformly Lipschitz continuous, with

$$
\begin{gathered}
\left\|J_{n} u-J_{n} v\right\| \leq\|u-v\|, \\
\left\|A_{n} u-A_{n} v\right\| \leq 2 n\|u-v\| .
\end{gathered}
$$

LEMMA 2.2. Let $A$ be $m$-monotonic. Then $A_{n}$ are also monotonic. Furthermore,

$$
\left\|A_{n} u\right\| \leq\|A u\|, \quad \text { for } u \in D(A)
$$

LEMMA 2.3. Let $A$ be $m$-monotonic in $H$.

(a) If $X^{n} \in D(A), n=1,2, \ldots, X^{n} \rightarrow u \in H$, and if $\left\|A X^{n}\right\|$ are bounded, then $u \in D(A)$ and $A X_{n} \rightarrow A u$.

(b) If $x_{n} \in H, n=1,2, \ldots, x_{n} \rightarrow u \in H$, and if $\left\|A_{n} x_{n}\right\|$ are bounded, then $u \in D(A)$ and $A_{n} x_{n} \rightarrow A u$.

Proof. (a) The monotonicity condition gives

$$
\left(A v-A X^{n}, v-X^{n}\right) \geq 0
$$

for any $v \in D(A)$. Since $H$ is reflexive and the $\left\|A X^{n}\right\|$ are bounded, there is a subsequence $\left\{X^{n^{\prime}}\right\}$ of $\left\{X^{n}\right\}$ such that $A X^{n^{\prime}}-w \in H$. Since $v-X^{n^{\prime}} \rightarrow v-u$, we obtain from (2.8) the inequality $(A v-w, v-u) \geq 0$. For fixed $z \in H, t>0$, put $v_{t}=A_{1}^{-1}(u+w+t z)$, where $A_{1}=(I+A)$. It follows that $A v_{t}+v_{t}-u-w=t z$, hence $t\left(z, v_{t}-u\right) \geq 0$ so $\left(z, v_{t}-u\right) \geq 0$. Letting $t \rightarrow 0, v_{t} \rightarrow A_{1}^{-1}(u+w)$, hence $\left(z, A_{1}^{-1}(u+w)-u\right) \geq 0$, so $u=A_{1}^{-1}(u+w), u \in D(A)$, and $A u=w$. It shows that $A X^{n}-w=A u$.

(b) Set $X^{n}=J_{n} x_{n} \in D(A)$. Then $A X^{n}=\left(A J_{n} x_{n}\right)=A_{n} x_{n}$ and $\left\|A X^{n}\right\|$ are bounded. Also, $x_{n}-X^{n}=\left(I-J_{n}\right) x_{n}=n^{-1} A_{n} x_{n} \rightarrow 0$, so that $X^{n} \rightarrow u$. Thus, by the result of (a), we get $u \in D(A)$ and $A_{n} x_{n}=A X^{n} \rightarrow A u$.

Now, we state the following main result of this paper which will be proved in the next section after proving some a priori estimates.

THEOREM 2.4. Suppose that the conditions (A1), (A2), (A3), (A4) are satisfied. Then (1.1) has a strong solution $u \in \mathscr{C}_{T}$ either on $[-\tau, T]$ or on the maximal interval of existence $\left[-\tau, t_{\max }\right), 0<t_{\max } \leq T$, and in the later case, either $\lim _{t \rightarrow t_{\max }-}\|u(t)\|=\infty$ or $u(t)$ goes to the boundary of $D(A)$ as $t \rightarrow t_{\max }-$. Moreover, $u$ is Lipschitz continuous on every compact subinterval of existence. 
3. Discretization scheme and a priori estimates. In this section, we establish the existence and uniqueness of a strong solution to (1.4) for any given $\chi \in \mathscr{C}_{T}$ with $\chi(0) \in$ $D(A)$. For the application of the method of lines to (1.4), we proceed as follows. Let $R_{0}:=\sup _{t \in[-\tau, T]}\|\chi(t)-\chi(0)\|$. For any $0<R \leq R_{0}$, we choose $t_{0}$ such that

$$
0<t_{0} \leq T, \quad t_{0}\left[\|A \chi(0)\|+3 L_{f}\left(R_{0}\right)\left(T+(m+1) R_{0}\right)+\|f(0, \chi(0), \chi(0), \ldots, \chi(0))\|\right] \leq R .
$$

For $n \in \mathbb{N}$, let $h_{n}=t_{0} / n$. We set $u_{0}^{n}=\chi(0)$ for all $n \in \mathbb{N}$ and define each of $\left\{u_{j}^{n}\right\}_{j=1}^{n}$ as the unique solution of the equation

$$
\frac{u-u_{j-1}^{n}}{h_{n}}+A u=f\left(t_{j}^{n}, u_{j-1}^{n}, \tilde{u}_{j-1}^{n}\left(r_{1}\left(t_{j}^{n}\right)\right), \ldots, \tilde{u}_{j-1}^{n}\left(r_{m}\left(t_{j}^{n}\right)\right)\right),
$$

where $\tilde{u}_{0}^{n}(t)=\chi(t)$ for $t \in[-\tau, 0], \tilde{u}_{0}^{n}(t)=\chi(0)$ for $t \in\left[0, t_{0}\right]$ and for $2 \leq j \leq n$,

$$
\tilde{u}_{j-1}^{n}(t)= \begin{cases}x(t), & t \in[-\tau, 0], \\ u_{i-1}^{n}+\frac{1}{h_{n}}\left(t-t_{i-1}^{n}\right)\left(u_{i}^{n}-u_{i-1}^{n}\right), & t \in\left[t_{i-1}^{n}, t_{i}^{n}\right], i=1,2, \ldots, j-1, \\ u_{j-1}^{n}, & t \in\left[t_{j-1}^{n}, t_{0}\right] .\end{cases}
$$

The existence of a unique $u_{j}^{n} \in D(A)$ satisfying (3.2) is a consequence of the $m$ monotonicity of $A$. Using (A2), we first prove that the points $\left\{u_{j}^{n}\right\}_{j=0}^{n}$ lie in a ball with its radius independent of the discretization parameters $j, h_{n}$, and $n$. We then prove a priori estimates on the difference quotients $\left\{u_{j}^{n}-u_{j-1}^{n} / h_{n}\right\}$ using (A2). We define the sequence $\left\{U^{n}\right\} \subset \mathscr{C}_{T}$ of polygonal functions

$$
U^{n}(t)= \begin{cases}x(t), & t \in[-\tau, 0], \\ u_{j-1}^{n}+\frac{1}{h_{n}}\left(t-t_{j-1}^{n}\right)\left(u_{j}^{n}-u_{j-1}^{n}\right), & t \in\left(t_{j-1}^{n}, t_{j}^{n}\right],\end{cases}
$$

and prove the convergence of $\left\{U^{n}\right\}$ to a unique strong solution $u$ of (1.4) in $\mathscr{C}_{T}$ as $n \rightarrow \infty$.

Now, we first show that $\left\{u_{j}^{n}\right\}_{j=0}^{n}$ lie in a ball in $H$ of radius independent of $j, h_{n}$, and $n$.

LEMMA 3.1. For $n \in \mathbb{N}, j=1,2, \ldots, n$,

$$
\left\|u_{j}^{n}-\chi(0)\right\| \leq R
$$

Proof. From (3.2) for $j=1$, we have

$$
\frac{u_{1}^{n}-u_{0}^{n}}{h_{n}}+A u_{1}^{n}=f\left(t_{1}^{n}, u_{0}^{n}, \tilde{u}_{0}^{n}\left(r_{1}\left(t_{1}^{n}\right)\right), \ldots, \tilde{u}_{0}^{n}\left(r_{m}\left(t_{1}^{n}\right)\right)\right) .
$$

Subtracting $A u_{0}^{n}$ on both sides, we get

$$
\frac{u_{1}^{n}-u_{0}^{n}}{h_{n}}+A u_{1}^{n}-A u_{0}^{n}=-A u_{0}^{n}+f\left(t_{1}^{n}, u_{0}^{n}, \tilde{u}_{0}^{n}\left(r_{1}\left(t_{1}^{n}\right)\right), \ldots, \tilde{u}_{0}^{n}\left(r_{m}\left(t_{1}^{n}\right)\right)\right)
$$


Taking the inner product with $u_{1}^{n}-u_{0}^{n}$, we get

$$
\begin{aligned}
& \left(\frac{u_{1}^{n}-u_{0}^{n}}{h_{n}}, u_{1}^{n}-u_{0}^{n}\right)+\left(A u_{1}^{n}-A u_{0}^{n}, u_{1}^{n}-u_{0}^{n}\right) \\
& \quad=-\left(A u_{0}^{n}, u_{1}^{n}-u_{0}^{n}\right)+\left(f\left(t_{1}^{n}, u_{0}^{n}, \tilde{u}_{0}^{n}\left(r_{1}\left(t_{1}^{n}\right)\right), \ldots, \tilde{u}_{0}^{n}\left(r_{m}\left(t_{1}^{n}\right)\right)\right), u_{1}^{n}-u_{0}^{n}\right) .
\end{aligned}
$$

By the monotonicity of $A$, we have

$$
\left\|u_{1}^{n}-u_{0}^{n}\right\| \leq h_{n}\left[\left\|A u_{0}^{n}\right\|+\left\|f\left(t_{1}^{n}, u_{0}^{n}, \tilde{u}_{0}^{n}\left(r_{1}\left(t_{1}^{n}\right)\right), \ldots, \tilde{u}_{0}^{n}\left(r_{m}\left(t_{1}^{n}\right)\right)\right)\right\|\right]
$$

Using (A3), we get

$$
\left\|u_{1}^{n}-\chi(0)\right\| \leq h_{n}\left[\|A \chi(0)\|+3 L_{f}\left(R_{0}\right)\left(T+(m+1) R_{0}\right)+\|f(0, \chi(0), \ldots, \chi(0))\|\right] \leq R
$$

Assume that $\left\|u_{i}^{n}-\chi(0)\right\| \leq R$ for $i=1,2, \ldots, j-1$.

Now, for $2 \leq j \leq n$,

$$
\frac{u_{j}^{n}-u_{j-1}^{n}}{h_{n}}+A u_{j}^{n}=f\left(t_{j}^{n}, u_{j-1}^{n}, \tilde{u}_{j-1}^{n}\left(r_{1}\left(t_{j}^{n}\right)\right), \ldots, \tilde{u}_{j-1}^{n}\left(r_{m}\left(t_{j}^{n}\right)\right)\right) .
$$

Subtracting $A \chi(0)$ on both sides, we get

$$
\frac{u_{j}^{n}-u_{j-1}^{n}}{h_{n}}+A u_{j}^{n}-A \chi(0)=-A \chi(0)+f\left(t_{j}^{n}, u_{j-1}^{n}, \tilde{u}_{j-1}^{n}\left(r_{1}\left(t_{j}^{n}\right)\right), \ldots, \tilde{u}_{j-1}^{n}\left(r_{m}\left(t_{j}^{n}\right)\right)\right) \text {. }
$$

Taking the inner product with $u_{j}^{n}-\chi(0)$, we get

$$
\begin{aligned}
& \frac{1}{h_{n}}\left(u_{j}^{n}-u_{j-1}^{n}, u_{j}^{n}-\chi(0)\right)+\left(A u_{j}^{n}-A \chi(0), u_{j}^{n}-\chi(0)\right) \\
& \quad=-\left(A \chi(0), u_{j}^{n}-\chi(0)\right)+\left(f\left(t_{j}^{n}, u_{j-1}^{n}, \tilde{u}_{j-1}^{n}\left(r_{1}\left(t_{j}^{n}\right)\right), \ldots, \tilde{u}_{j-1}^{n}\left(r_{m}\left(t_{j}^{n}\right)\right)\right), u_{j}^{n}-\chi(0)\right) .
\end{aligned}
$$

By the monotonicity of $A$, we have

$$
\begin{aligned}
\left\|u_{j}^{n}-\chi(0)\right\| \leq & \left\|u_{j-1}^{n}-\chi(0)\right\| \\
& +h_{n}\left[\|A \chi(0)\|+\left\|f\left(t_{j}^{n}, u_{j-1}^{n}, \tilde{u}_{j-1}^{n}\left(r_{1}\left(t_{j}^{n}\right)\right), \ldots, \tilde{u}_{j-1}^{n}\left(r_{m}\left(t_{j}^{n}\right)\right)\right)\right\|\right] .
\end{aligned}
$$

Using (A3), we get

$$
\begin{aligned}
\left\|u_{j}^{n}-\chi(0)\right\| \leq & \left\|u_{j-1}^{n}-\chi(0)\right\| \\
& +h_{n}\left[\|A \chi(0)\|+3 L_{f}\left(R_{0}\right)\left(T+(m+1) R_{0}\right)+\|f(0, \chi(0), \chi(0), \ldots, \chi(0))\|\right] .
\end{aligned}
$$


Repeating the above inequality, we obtain

$$
\begin{array}{r}
\left\|u_{j}^{n}-\chi(0)\right\| \leq j h_{n}\left[\|A \chi(0)\|+3 L_{f}\left(R_{0}\right)\left(T+(m+1) R_{0}\right)\right. \\
+\|f(0, \chi(0), \chi(0), \ldots, \chi(0))\|] \leq R,
\end{array}
$$

as $j h_{n} \leq t_{0}$ for $0 \leq j \leq n$. This completes the proof of the lemma.

Now, we establish a priori estimates for the difference quotients $\left\{\left(u_{j}^{n}-u_{j-1}^{n}\right) / h_{n}\right\}$.

LEMMA 3.2. There exists a positive constant $K$ independent of the discretization parameters $n, j$, and $h_{n}$ such that

$$
\left\|\frac{u_{j}^{n}-u_{j-1}^{n}}{h_{n}}\right\| \leq K, \quad j=1,2, \ldots, n, n=1,2, \ldots .
$$

Proof. In this proof and subsequently, $K$ will represent a generic constant independent of $j, h_{n}$, and $n$. Subtracting $A u_{0}^{n}=A \chi(0)$ from both sides in (3.2), we get

$$
\frac{u_{1}^{n}-u_{0}^{n}}{h_{n}}+A u_{1}^{n}-A u_{0}^{n}=-A u_{0}^{n}+f\left(t_{1}^{n}, u_{0}^{n}, \tilde{u}_{0}^{n}\left(r_{1}\left(t_{1}^{n}\right)\right), \ldots, \tilde{u}_{0}^{n}\left(r_{m}\left(t_{1}^{n}\right)\right)\right) .
$$

Taking the inner product with $\left(u_{1}^{n}-u_{0}^{n}\right)$, we get

$$
\begin{aligned}
& \left(\frac{u_{1}^{n}-u_{0}^{n}}{h_{n}}, u_{1}^{n}-u_{0}^{n}\right)+\left(A u_{1}^{n}-A u_{0}^{n}, u_{1}^{n}-u_{0}^{n}\right) \\
& \quad=-\left(A u_{0}^{n}, u_{1}^{n}-u_{0}^{n}\right)+\left(f\left(t_{1}^{n}, u_{0}^{n}, \tilde{u}_{0}^{n}\left(r_{1}\left(t_{1}^{n}\right)\right), \ldots, \tilde{u}_{0}^{n}\left(r_{m}\left(t_{1}^{n}\right)\right)\right), u_{1}^{n}-u_{0}^{n}\right) .
\end{aligned}
$$

Using monotonicity of $A$, we get

$$
\left\|\frac{u_{1}^{n}-u_{0}^{n}}{h_{n}}\right\| \leq\|A \chi(0)\|+\left\|f\left(t_{1}^{n}, u_{0}^{n}, \tilde{u}_{0}^{n}\left(r_{1}\left(t_{1}^{n}\right)\right), \ldots, \tilde{u}_{0}^{n}\left(r_{m}\left(t_{1}^{n}\right)\right)\right)\right\| .
$$

Using (A3), we have

$$
\begin{aligned}
\left\|\frac{u_{1}^{n}-u_{0}^{n}}{h_{n}}\right\| & \leq\|A \chi(0)\|+\|f(0, \chi(0), \chi(0), \ldots, \chi(0))\|+3 L_{f}\left(R_{0}\right)\left(T+(m+1) R_{0}\right) \\
& \leq K
\end{aligned}
$$

Now, for $2 \leq j \leq n$, taking the inner product with $\left(u_{j}^{n}-u_{j-1}^{n}\right)$ in (3.2), we get

$$
\begin{aligned}
& \left(\frac{u_{j}^{n}-u_{j-1}^{n}}{h_{n}}, u_{j}^{n}-u_{j-1}^{n}\right)+\left(A u_{j}^{n}, u_{j}^{n}-u_{j-1}^{n}\right) \\
& \quad=\left(f\left(t_{j}^{n}, u_{j-1}^{n}, \tilde{u}_{j-1}^{n}\left(r_{1}\left(t_{j}^{n}\right)\right), \ldots, \tilde{u}_{j-1}^{n}\left(r_{m}\left(t_{j}^{n}\right)\right)\right), u_{j}^{n}-u_{j-1}^{n}\right) .
\end{aligned}
$$

Also,

$$
\begin{aligned}
\left(\frac{u_{j}^{n}-u_{j-1}^{n}}{h_{n}}-\frac{u_{j-1}^{n}-u_{j-2}^{n}}{h_{n}}, u_{j}^{n}-u_{j-1}^{n}\right)+\left(A u_{j}^{n}-A u_{j-1}^{n}, u_{j}^{n}-u_{j-1}^{n}\right) \\
=\left(f\left(t_{j}^{n}, u_{j-1}^{n}, \tilde{u}_{j-1}^{n}\left(r_{1}\left(t_{j}^{n}\right)\right), \ldots, \tilde{u}_{j-1}^{n}\left(r_{m}\left(t_{j}^{n}\right)\right)\right)\right. \\
\left.\quad-f\left(t_{j-1}^{n}, u_{j-2}^{n}, \tilde{u}_{j-2}^{n}\left(r_{1}\left(t_{j-1}^{n}\right)\right), \ldots, \tilde{u}_{j-2}^{n}\left(r_{m}\left(t_{j-1}^{n}\right)\right)\right), u_{j}^{n}-u_{j-1}^{n}\right) .
\end{aligned}
$$


Using the monotonicity of $A$, we get

$$
\begin{aligned}
\left\|\frac{u_{j}^{n}-u_{j-1}^{n}}{h_{n}}\right\| \leq & \left\|\frac{u_{j-1}^{n}-u_{j-2}^{n}}{h_{n}}\right\| \\
+ & \| f\left(t_{j}^{n}, u_{j-1}^{n}, \tilde{u}_{j-1}^{n}\left(r_{1}\left(t_{j}^{n}\right)\right), \ldots, \tilde{u}_{j-1}^{n}\left(r_{m}\left(t_{j}^{n}\right)\right)\right) \\
& -f\left(t_{j-1}^{n}, u_{j-2}^{n}, \tilde{u}_{j-2}^{n}\left(r_{1}\left(t_{j-1}^{n}\right)\right), \ldots, \tilde{u}_{j-2}^{n}\left(r_{m}\left(t_{j-1}^{n}\right)\right)\right) \| .
\end{aligned}
$$

Now,

$$
\begin{aligned}
& \| f\left(t_{j}^{n}, u_{j-1}^{n}, \tilde{u}_{j-1}^{n}\left(r_{1}\left(t_{j}^{n}\right)\right), \ldots, \tilde{u}_{j-1}^{n}\left(r_{m}\left(t_{j}^{n}\right)\right)\right) \\
& -f\left(t_{j-1}^{n}, u_{j-2}^{n}, \tilde{u}_{j-2}^{n}\left(r_{1}\left(t_{j-1}^{n}\right)\right), \ldots, \tilde{u}_{j-2}^{n}\left(r_{m}\left(t_{j-1}^{n}\right)\right)\right) \| \\
& \quad \leq C\left[h_{n}+(m+1) h_{n}\left\|\frac{u_{j}^{n}-u_{j-1}^{n}}{h_{n}}\right\|\right] .
\end{aligned}
$$

From the above inequality, we get

$$
\begin{aligned}
\max _{\{1 \leq k \leq j\}}\left\|\frac{u_{k}^{n}-u_{k-1}^{n}}{h_{n}}\right\| & \leq\left(1+C h_{n}\right) \max _{\{1 \leq k \leq j-1\}}\left\|\frac{u_{k}^{n}-u_{k-1}^{n}}{h_{n}}\right\|+C h_{n} \\
& \leq\left(1+C h_{n}\right)\left[1+\max _{\{1 \leq k \leq j-1\}}\left\|\frac{u_{k}^{n}-u_{k-1}^{n}}{h_{n}}\right\|\right],
\end{aligned}
$$

where $C$ is a positive constant independent of $j, h_{n}$, and $n$. Repeating the above inequality, we get

$$
\max _{\{1 \leq k \leq j\}}\left\|\frac{u_{j}^{n}-u_{j-1}^{n}}{h_{n}}\right\| \leq\left(1+C h_{n}\right)^{j} D \leq D e^{T C} \leq K .
$$

This completes the proof of the lemma.

We introduce another sequence $\left\{X^{n}\right\}$ of step functions from $[0, T]$ into $H$ by

$$
X^{n}(t)= \begin{cases}x(0), & t=0, \\ u_{j}^{n}, & t \in\left(t_{j-1}^{n}, t_{j}^{n}\right] .\end{cases}
$$

REMARK 3.3. From Lemma 3.2, it follows that the functions $U^{n}$ and $\tilde{u}_{r}^{n}, 0 \leq r \leq n-1$, are Lipschitz continuous on $\left[0, t_{0}\right]$ with a uniform Lipschitz constant $K$. The sequence $U^{n}(t)-X^{n}(t) \rightarrow 0$ in $H$ as $n \rightarrow \infty$ uniformly on [0, $\left.t_{0}\right]$. Furthermore, $X^{n}(t) \in D(A)$ for $t \in\left[0, t_{0}\right]$ and the sequences $\left\{U^{n}(t)\right\}$ and $\left\{X^{n}(t)\right\}$ are bounded in $H$, uniformly in $n \in \mathbb{N}$ and $t \in\left[0, t_{0}\right]$. The sequence $\left\{A X^{n}(t)\right\}$ is bounded uniformly in $n \in \mathbb{N}$ and $t \in\left[0, t_{0}\right]$.

For notational convenience, let

$$
f^{n}(t)=f\left(t_{j}^{n}, u_{j-1}^{n}, \tilde{u}_{j-1}^{n}\left(r_{1}\left(t_{j}^{n}\right)\right), \ldots, \tilde{u}_{j-1}^{n}\left(r_{m}\left(t_{j}^{n}\right)\right)\right), \quad t \in\left(t_{j-1}^{n}, t_{j}^{n}\right], 1 \leq j \leq n .
$$


Then (3.2) may be rewritten as

$$
\frac{d^{-}}{d t} U^{n}(t)+A X^{n}(t)=f^{n}(t), \quad t \in\left(0, t_{0}\right]
$$

where $d^{-} / d t$ denotes the left derivative in $\left(0, t_{0}\right]$. Also, for $t \in\left(0, t_{0}\right]$, we have

$$
\int_{0}^{t} A X^{n}(s) d s=\chi(0)-U^{n}(t)+\int_{0}^{t} f^{n}(s) d s .
$$

LEMmA 3.4. There exists $u \in \mathscr{C}_{t_{0}}$ such that $U^{n} \rightarrow u$ in $\mathscr{C}_{t_{0}}$ as $n \rightarrow \infty$. Moreover, $u$ is Lipschitz continuous on $\left[0, t_{0}\right]$.

Proof. From (3.30) for $t \in\left(0, t_{0}\right]$, and taking the inner product with $\left(X^{n}(t)-X^{k}(t)\right)$, we have

$$
\begin{gathered}
\left(\frac{d^{-}}{d t} U^{n}(t)-\frac{d^{-}}{d t} U^{k}(t), X^{n}(t)-X^{k}(t)\right) \\
+\left(A X^{n}(t)-A X^{k}(t), X^{n}(t)-X^{k}(t)\right) \\
=\left(f^{n}(t)-f^{k}(t), X^{n}(t)-X^{k}(t)\right) .
\end{gathered}
$$

Using monotonicity of $A$, we have

$$
\left(\frac{d^{-}}{d t}\left(U^{n}(t)-U^{k}(t)\right), X^{n}(t)-X^{k}(t)\right) \leq\left(f^{n}(t)-f^{k}(t), X^{n}(t)-X^{k}(t)\right) .
$$

From the above inequality and the fact that

$$
2\left(\frac{d^{-}}{d t} U^{n}(t)-\frac{d^{-}}{d t} U^{k}(t), U^{n}(t)-U^{k}(t)\right)=\frac{d^{-}}{d t}\left\|U^{n}(t)-U^{k}(t)\right\|^{2},
$$

we get

$$
\begin{aligned}
\frac{1}{2} \frac{d^{-}}{d t} \| & U^{n}(t)-U^{k}(t) \|^{2} \\
\leq & \left(\frac{d^{-}}{d t}\left(U^{n}(t)-U^{k}(t)\right)+f^{n}(t)-f^{k}(t)-f^{n}(t)+f^{k}(t),\right. \\
& \left.U^{n}(t)-U^{k}(t)-X^{n}(t)+X^{k}(t)\right)+\left(f^{n}(t)-f^{k}(t), X^{n}(t)-X^{k}(t)\right) \\
= & \left(\frac{d^{-}}{d t}\left(U^{n}(t)-U^{k}(t)\right)-f^{n}(t)+f^{k}(t), U^{n}(t)-U^{k}(t)-X^{n}(t)+X^{k}(t)\right) \\
+ & \left(f^{n}(t)-f^{k}(t), U^{n}(t)-U^{k}(t)\right) .
\end{aligned}
$$

Now,

$$
\begin{aligned}
\left\|f^{n}(t)-f^{k}(t)\right\|= & \| f\left(t_{j}^{n}, u_{j-1}^{n}, \tilde{u}_{j-1}^{n}\left(r_{1}\left(t_{j}^{n}\right)\right), \ldots, \tilde{u}_{j-1}^{n}\left(r_{m}\left(t_{j}^{n}\right)\right)\right) \\
& -f\left(t_{l}^{k}, u_{l-1}^{k}, \tilde{u}_{l-1}^{k}\left(r_{1}\left(t_{l}^{k}\right)\right), \ldots, \tilde{u}_{l-1}^{k}\left(r_{m}\left(t_{l}^{k}\right)\right)\right) \| .
\end{aligned}
$$

Using (A3), we get

$$
\left\|f^{n}(t)-f^{k}(t)\right\| \leq \epsilon_{n k}(t)+K\left\|U^{n}-U^{k}\right\|_{t}
$$


where

$$
\begin{aligned}
\epsilon_{n k}(t)=K[ & h_{n}+h_{k}+\left|t_{j}^{n}-t_{l}^{k}\right|+\left\|X^{n}\left(t-h_{n}\right)-U^{n}(t)\right\| \\
& +\left\|X^{k}\left(t-h_{k}\right)-U^{k}(t)\right\| \\
& \left.+\sum_{i=1}^{m}\left(\left|r_{i}(t)-r_{i}\left(t_{j}^{n}\right)\right|+\left|r_{i}(t)-r_{i}\left(t_{l}^{k}\right)\right|\right)\right],
\end{aligned}
$$

for $t \in\left(t_{j-1}^{n}, t_{j}^{n}\right]$ and $t \in\left(t_{l-1}^{k}, t_{l}^{k}\right], 1 \leq j \leq n, 1 \leq l \leq k$. Therefore $\epsilon_{n k}(t) \rightarrow 0$ as $n, k \rightarrow \infty$ uniformly on $\left[0, t_{0}\right]$. This implies that for a.e. $t \in\left[0, t_{0}\right]$,

$$
\begin{aligned}
\frac{d^{-}}{d t} \| U^{n}( & t)-U^{k}(t) \|^{2} \\
\leq 2[ & \left\|\frac{d^{-}}{d t}\left(U^{n}(t)-U^{k}(t)-f^{n}(t)-f^{k}(t)\right)\right\| \\
\times & \left\|U^{n}(t)-U^{k}(t)-X^{n}(t)+X^{k}(t)\right\| \\
& \left.+\left\|f^{n}(t)-f^{k}(t)\right\|\left\|U^{n}(t)-U^{k}(t)\right\|\right] \\
\leq K & {\left[\epsilon_{n k}+\left\|U^{n}-U^{k}\right\|_{t}^{2}\right], }
\end{aligned}
$$

where $\epsilon_{n k}$ is a sequence of numbers such that $\epsilon_{n k} \rightarrow 0$ as $n, k \rightarrow \infty$. Integrating the above inequality over $(0, s), 0<s \leq t \leq t_{0}$, and using the fact that $U^{n}=\phi$ on $[-\tau, 0]$ for all $n$, we get

$$
\left\|U^{n}(s)-U^{k}(s)\right\|^{2} \leq K\left[T \epsilon_{n k}+\int_{0}^{t}\left\|U^{n}-U^{k}\right\|_{s}^{2} d s\right]
$$

Taking the supremum over $[0, t]$, we get

$$
\sup _{s \in[0, t]}\left\|U^{n}(s)-U^{k}(s)\right\|^{2} \leq K\left[T \epsilon_{n k}+\int_{0}^{t}\left\|U^{n}-U^{k}\right\|_{s}^{2} d s\right] .
$$

Thus,

$$
\left\|U^{n}-U^{k}\right\|_{t}^{2} \leq K\left[T \epsilon_{n k}+\int_{0}^{t}\left\|U^{n}-U^{k}\right\|_{s}^{2} d s\right]
$$

Applying Gronwall's inequality, we conclude that there exists $u \in \mathscr{C}_{t_{0}}$ such that $U^{n} \rightarrow u$ in $\mathscr{C}_{t_{0}}$. Clearly, $u=\phi$ on $[-\tau, 0]$ and, from Remark 3.3, it follows that $u$ is Lipschitz continuous on $\left[0, t_{0}\right]$. This completes the proof of the lemma.

Proof of Theorem 2.4. We first prove the existence on $\left[-\tau, t_{0}\right]$ and then prove the unique continuation of the solution on $[-\tau, T]$. From Lemma 2.3(a), we know that $u(t) \in D(A)$ for $t \in\left[0, t_{0}\right], A X^{n}(t) \rightarrow A u(t)$ on $\left[0, t_{0}\right]$. Here, $\rightarrow$ denotes the weak convergence in $H$. Also, we may show that $A u(t)$ is weakly continuous on $\left[0, t_{0}\right]$ as follows.

Let $t_{i} \rightarrow t ; t_{i}, t \in\left[0, t_{0}\right]$, then $u\left(t_{i}\right) \rightarrow u(t)$ and, by boundedness of $A\left(u\left(t_{i}\right)\right)$ and reflexivity of $H$, there exists a sequence $\left\{u\left(t_{j}\right)\right\}$ of $\left\{u\left(t_{i}\right)\right\}$ such that $A u\left(t_{j}\right) \rightarrow w(t)$. Then it follows from Lemma 2.3(a) that $A u\left(t_{i}\right) \rightarrow A u(t)$. 
For every $x^{*} \in X^{*}$ and $t \in\left(0, t_{0}\right]$, we have

$$
\int_{0}^{t}\left\langle A X^{n}(s), x^{*}\right\rangle d s=\left\langle x(0), x^{*}\right\rangle-\left\langle U^{n}(t), x^{*}\right\rangle+\int_{0}^{t}\left\langle f^{n}(s), x^{*}\right\rangle d s .
$$

Using Lemma 3.4 and the bounded convergence theorem, we obtain as $n \rightarrow \infty$,

$$
\begin{aligned}
\int_{0}^{t}\left\langle A u(s), x^{*}\right\rangle d s= & \left\langle x(0), x^{*}\right\rangle-\left\langle u(t), x^{*}\right\rangle \\
& +\int_{0}^{t}\left\langle f\left(s, u(s), u\left(r_{1}(s)\right), \ldots, u\left(r_{m}(s)\right)\right), x^{*}\right\rangle d s .
\end{aligned}
$$

If $A u(t)$ is Bochner integrable (cf. [2]) on [0, $\left.t_{0}\right]$, from (3.44), we get

$$
\frac{d}{d t} u(t)+A u(t)=f\left(t, u(t), u\left(r_{1}(t)\right), \ldots, u\left(r_{m}(t)\right)\right), \quad \text { a.e. } t \in\left[0, t_{0}\right]
$$

To show that $A u(t)$ is Bochner integrable, we will use Lemmas 2.1 and 2.2 which state that operators $J_{n}$ and $A_{n}$ are Lipschitz continuous. Also,

$$
\left\|A_{n} u\right\| \leq\|A u\|, \quad \text { for } u \in D(A)
$$

Now,

$$
\begin{aligned}
\left\|A_{n} U^{n}(t)\right\| & \leq\left\|A_{n} U^{n}(t)-A_{n} X^{n}(t)\right\|+\left\|A_{n} X^{n}(t)\right\| \\
& \leq 2 n\left\|U^{n}(t)-X^{n}(t)\right\|+\left\|A X^{n}(t)\right\| \\
& \leq K
\end{aligned}
$$

where $K$ is a constant.

Then Lemma 2.3(b) implies that $A_{n} U^{n}(t)-A u(t)$.

Let $H_{0}$ be the smallest closed linear subspace of $H$ containing all the values of the $A_{n} U^{n}(t)$ for $t \in\left[0, t_{0}\right]$ and $n=1,2, \ldots$. Since $A_{n} U^{n}(t)$ are continuous, $H_{0}$ is separable. Since $A_{n} U^{n}(t)-A u(t)$ and $H_{0}$ is weakly closed, $A u(t) \in H_{0}$ too. Thus $A u(t)$ is separably valued. Since it is weakly continuous, it is strongly measurable and, being bounded, it is Bochner integrable (see [12]).

Clearly, $u$ is Lipschitz continuous on $\left[0, t_{0}\right]$ and $u(t) \in D(A)$ for $t \in\left[0, t_{0}\right]$. Now we prove the uniqueness of a function $u \in \mathscr{C}_{t_{0}}$ which is differentiable a.e. on $\left[0, t_{0}\right]$ with $u(t) \in D(A)$ a.e. on $\left[0, t_{0}\right]$ and $u=\phi$ on $[-\tau, 0]$ satisfying (3.45). Let $u_{1}, u_{2} \in \mathscr{C}_{t_{0}}$ be two such functions. Let

$$
R=\max \left\{\left\|u_{1}\right\|_{t_{0}},\left\|u_{2}\right\|_{t_{0}}\right\}
$$

Then for $u=u_{1}-u_{2}$, we have

$$
\begin{aligned}
&\left(\frac{d u}{d t}(t), u(t)\right)+\left(A u_{1}(t)-A u_{2}(t), u(t)\right) \\
&=( f\left(t, u_{1}(t), u_{1}\left(r_{1}(t)\right), \ldots, u_{1}\left(r_{m}(t)\right)\right) \\
&\left.\quad-f\left(t, u_{2}(t), u_{2}\left(r_{1}(t)\right), \ldots, u_{2}\left(r_{m}(t)\right)\right), u(t)\right) .
\end{aligned}
$$


By the monotonicity of $A$,

$$
\begin{aligned}
\frac{d}{d t}\|u(t)\|^{2} \leq 2 \| & f\left(t, u_{1}(t), u_{1}\left(r_{1}(t)\right), \ldots, u_{1}\left(r_{m}(t)\right)\right) \\
& -f\left(t, u_{2}(t), u_{2}\left(r_{1}(t)\right), \ldots, u_{2}\left(r_{m}(t)\right)\right)\|\| u(t) \| .
\end{aligned}
$$

It implies that

$$
\frac{d}{d t}\|u(t)\|^{2} \leq C(R)\|u\|_{t}^{2} \text {, a.e. } t \in\left[0, t_{0}\right]
$$

where $C: \mathbb{R}_{+} \rightarrow \mathbb{R}_{+}$is a nondecreasing function. Integrating over $(0, s)$ for $0<s \leq t \leq t_{0}$, and using the fact that $u \equiv 0$ on $[-\tau, 0]$, we get

$$
\|u(s)\|^{2} \leq C(R) \int_{0}^{t}\|u\|_{s}^{2} d s
$$

Taking the supremum over $[0, t]$, we get

$$
\sup _{s \in[0, t]}\|u(s)\|^{2} \leq C(R) \int_{0}^{t}\|u\|_{s}^{2} d s .
$$

Thus,

$$
\|u\|_{t}^{2} \leq C(R) \int_{0}^{t}\|u\|_{s}^{2} d s
$$

Application of Gronwall's inequality implies that $u \equiv 0$ on $\left[-\tau, t_{0}\right]$.

Now, we prove the continuation of the solution $u$ on $[-\tau, T]$. Suppose $t_{0}<T$ and consider the problem

$$
\begin{gathered}
w^{\prime}(t)+A w(t)=\tilde{f}\left(t, w(t), w\left(\tilde{r}_{1}(t)\right), w\left(\tilde{r}_{2}(t)\right), \ldots, w\left(\tilde{r}_{m}(t)\right)\right), \quad 0<t \leq T-t_{0}, \\
w=\tilde{x}, \quad \text { on }\left[-\tau-t_{0}, 0\right],
\end{gathered}
$$

where

$$
\begin{gathered}
\tilde{f}\left(t, u_{1}, u_{2}, \ldots, u_{m+1}\right)=f\left(t+t_{0}, u_{1}, u_{2}, \ldots, u_{m+1}\right), \quad 0 \leq t \leq T-t_{0}, \\
\tilde{x}(t)= \begin{cases}x\left(t+t_{0}\right), & t \in\left[-\tau-t_{0},-t_{0}\right], \\
u\left(t+t_{0}\right), & t \in\left[-t_{0}, 0\right],\end{cases} \\
\tilde{r}_{i}(t)=r_{i}\left(t+t_{0}\right)-t_{0}, \quad t \in\left[0, T-t_{0}\right], i=1,2, \ldots, m .
\end{gathered}
$$

Since $\tilde{x}(0)=u\left(t_{0}\right) \in D(A), \tilde{f}$ satisfies (A3), and $\tilde{r}_{i}, i=1,2, \ldots, m$, satisfy (A4) on $\left[0, T-t_{0}\right]$, we may proceed as before and prove the existence of a unique $w \in C([-\tau-$ $\left.\left.t_{0}, t_{1}\right] ; X\right), 0<t_{1} \leq T-t_{0}$, such that $w$ is Lipschitz continuous on $\left[0, t_{1}\right], w(t) \in D(A)$ for $t \in\left[0, t_{1}\right]$, and $w$ satisfies

$$
\begin{gathered}
w^{\prime}(t)+A w(t)=\tilde{f}\left(t, w(t), w\left(\tilde{r}_{1}(t)\right), w\left(\tilde{r}_{2}(t)\right), \ldots, w\left(\tilde{r}_{m}(t)\right)\right), \quad \text { a.e. } t \in\left[0, t_{1}\right], \\
w=\tilde{x}, \quad \text { on }\left[-\tau-t_{0}, 0\right] .
\end{gathered}
$$


Then the function

$$
\bar{u}(t)= \begin{cases}u(t), & t \in\left[-\tau, t_{0}\right], \\ w\left(t-t_{0}\right), & t \in\left[t_{0}, t_{0}+t_{1}\right],\end{cases}
$$

is Lipschitz continuous on $\left[0, t_{0}+t_{1}\right], \bar{u}(t) \in D(A)$ for $t \in\left[0, t_{0}+t_{1}\right]$, and satisfies (1.4) a.e. on $\left[0, t_{1}\right]$. Continuing this way, we may prove the existence either on the whole interval $[-\tau, T]$ or on the maximal interval of existence $\left[-\tau, t_{\max }\right), 0<t_{\max } \leq T$. In case $\lim _{t \rightarrow t_{\max }-}\|u(t)\|<\infty$, then as $u(t) \in D(A)$ for $t \in\left[0, t_{\max }\right)$, we have that $\lim _{t \rightarrow t_{\max }-} u(t)$ is in the closure of $D(A)$ in $H$, and if it is in $D(A)$, then proceeding as before, we may extend $u(t)$ beyond $t_{\max }$ contradicting the definition of the maximal interval of existence.

Now, let $u_{\chi}$ be the strong solution of (1.4) corresponding to $\chi \in \mathscr{C}_{\tilde{T}}$ satisfying $h\left(\chi_{[-\tau, 0]}\right)=\phi_{0}\left(\tilde{T}\right.$ is either equal to $T$ or $\left.\tilde{T}<t_{\max }\right)$. If there are $\chi^{1}, \chi^{2} \in \mathscr{C}_{\tilde{T}}$ such that $h\left(\chi_{[-\tau, 0]}^{1}\right)=h\left(\chi_{[-\tau, 0]}^{2}\right)=\phi_{0}$ and $\chi^{1} \neq \chi^{2}$ on $[-\tau, 0]$, then clearly $u_{\chi^{1}}$ and $u_{\chi^{2}}$, satisfying (1.4), are different. This completes the proof of Theorem 2.4.

4. Applications. Theorem 2.4 may be applied to get the existence and uniqueness results for (1.1) in the case when the operator $A$, with the domain $D(A)=H^{2 m}(\Omega) \cap$ $H_{0}^{m}(\Omega)$ into $H:=L^{2}(\Omega)$, is associated with the nonlinear partial differential operator

$$
A u=\sum_{|\alpha| \leq m}(-1)^{|\alpha|} D^{\alpha} A_{\alpha}\left(x, u(x), D u, \ldots, D^{\alpha} u\right)
$$

in a bounded $\Omega$ in $\mathbb{R}^{n}$ with sufficiently smooth boundary $\partial \Omega$, where $A_{\alpha}(x, \xi)$ are real functions defined on $\Omega \times \mathbb{R}^{N}$ for some $N \in \mathbb{N}$ and satisfying the following conditions.

(I) $A_{\alpha}$ are measurable in $x$ and continuous in $\xi$. There exist $p=2, g \in L^{2}(\Omega)$, and a positive constant $C$ such that

$$
\left|A_{\alpha}(x, \xi)\right| \leq C(|\xi|+g(x)), \quad \text { a.e. } x \in \Omega,
$$

where $\xi=\left(\xi_{\alpha} ;|\alpha| \leq m\right)$.

(II) For any $(\xi, \eta) \in \mathbb{R}^{N} \times \mathbb{R}^{N}$ and for almost every $x \in \Omega$, the following inequality holds:

$$
\sum_{|\alpha| \leq m}\left(A_{\alpha}(x, \xi)-A_{\alpha}(x, \eta)\right)\left(\xi_{\alpha}-\eta_{\alpha}\right) \geq 0
$$

In (1.1), we may take $f$ as the function $f:[0, T] \times\left(L^{2}(\Omega)\right)^{m+1} \rightarrow L^{2}(\Omega)$, given by

$$
f\left(t, u_{1}, u_{2}, \ldots, u_{m+1}\right)=f_{0}(t)+a(t) \sum_{i=1}^{m+1}\left\|u_{i}\right\|_{L^{2}(\Omega)} u_{i},
$$

where $f_{0}:[0, T] \rightarrow L^{2}(\Omega)$ and $a:[0, T] \rightarrow \mathbb{R}$ are Lipschitz continuous functions on $[0, T]$ and $\|\cdot\|_{L^{2}(\Omega)}$ denotes the norm in $L^{2}(\Omega)$. Let $\phi_{0} \in C\left([-\tau, 0] ; L^{2}(\Omega)\right)$ be such that $\phi_{0}(0) \in D(A)$. For the functions $r_{i}, i=1,2, \ldots, n$, and $h$, we may have any of the following. 
(b1) For $i=1,2, \ldots, m$, let $r_{i}(t)=t-\tau_{i}, t \in[0, T]$.

(b2) For $i=1,2, \ldots, m$, let $r_{i}(t)=k_{i} t, t \in[0, T], 0<k_{i} \leq 1$.

(b3) Let $T=1$ and $N_{0} \in \mathbb{N}$. For $i=1,2, \ldots, m$, for $t \in[0, T]$, let $r_{i}(t)=k_{i} t^{N_{0}}, 0<k_{i} \leq 1$.

(h1) For $u \in C\left([-\tau, 0] ; L^{2}(\Omega)\right)$, let

$$
g(u)=\int_{-\tau}^{0} k(s) u(s) d s, \quad \phi_{0}(t) \equiv u_{0}, \quad t \in[-\tau, 0], u_{0} \in D(A),
$$

where $\kappa=\int_{-\tau}^{0} k(s) d s \neq 0$. Let $h(u)(t) \equiv g(u)$ for $t \in[-\tau, 0]$. In this case, we may take $\chi(t) \equiv(1 / \kappa) u_{0}$ on $[-\tau, T]$.

(h2) For $u \in C\left([-\tau, 0] ; L^{2}(\Omega)\right),-\tau \leq a_{1}<a_{2}<\cdots<a_{r} \leq 0$, and $c_{i}>0, i=1,2, \ldots, r$, with $C:=\sum_{i=1}^{r} c_{i} \neq 0$, let

$$
g(u)=\sum_{i=1}^{r} c_{i} u\left(a_{i}\right),
$$

with $h$ and $\phi_{0}$ as in (h1). In this case, we may take $\chi(t) \equiv(1 / C) u_{0}$ for $t \in[-\tau, T]$. (h3) With $u, a_{i}$, and $c_{i}$ as in (h2) and $\epsilon_{i}>0$ for $i=1,2, \ldots, r$, let

$$
g(u)=\sum_{i}^{r} \frac{c_{i}}{\epsilon_{i}} \int_{a_{i}-\epsilon_{i}}^{a_{i}} u(s) d s,
$$

with $h, \phi_{0}$, and $u_{0}$ as in (h1). In this case, we may take $\chi(t)$ as in (h2).

ACKNOWLEDGMENTS. The authors would like to thank the National Board for Higher Mathematics for providing the financial support to carry out this work under its research project no. NBHM/2001/R\&D-II.

\section{REFERENCES}

[1] D. Bahuguna, Quasilinear integrodifferential equations in Banach spaces, Nonlinear Anal. 24 (1995), no. 2, 175-183.

[2] D. Bahuguna and V. Raghavendra, Application of Rothe's method to nonlinear Schrödinger type equations, Appl. Anal. 31 (1988), no. 1-2, 149-160.

[3] _ _ Application of Rothe's method to nonlinear integro-differential equations in Hilbert spaces, Nonlinear Anal. 23 (1994), no. 1, 75-81.

[4] K. Balachandran and M. Chandrasekaran, Existence of solutions of a delay differential equation with nonlocal condition, Indian J. Pure Appl. Math. 27 (1996), no. 5, 443-449.

[5] V. Barbu, Nonlinear Semigroups and Differential Equations in Banach Spaces, Noordhoff International Publishing, Leiden, 1976.

[6] L. Byszewski, Theorems about the existence and uniqueness of solutions of a semilinear evolution nonlocal Cauchy problem, J. Math. Anal. Appl. 162 (1991), no. 2, 494-505.

[7] L. Byszewski and V. Lakshmikantham, Theorem about the existence and uniqueness of a solution of a nonlocal abstract Cauchy problem in a Banach space, Appl. Anal. 40 (1991), no. 1, 11-19.

[8] A. G. Kartsatos, On the construction of methods of lines for functional evolutions in general Banach spaces, Nonlinear Anal. 25 (1995), no. 12, 1321-1331.

[9] A. G. Kartsatos and M. E. Parrott, A method of lines for a nonlinear abstract functional evolution equation, Trans. Amer. Math. Soc. 286 (1984), no. 1, 73-89.

[10] - A method of lines for a nonlinear abstract functional evolution equation, Trans. Amer. Math. Soc. 286 (1984), no. 1, 73-89. 
[11] Y. P. Lin and J. H. Liu, Semilinear integrodifferential equations with nonlocal Cauchy problem, Nonlinear Anal. 26 (1996), no. 5, 1023-1033.

[12] K. Yosida, Functional Analysis, Die Grundlehren der Mathematischen Wissenschaften, Band 123, Springer-Verlag, Berlin, 1965.

S. Agarwal: Department of Mathematics, Indian Institute of Technology, Kanpur 208016, India E-mail address: shrutiag@i itk.ac.in

D. Bahuguna: Department of Mathematics, Indian Institute of Technology, Kanpur 208016, India

E-mail address: dhiren@i itk.ac. in 


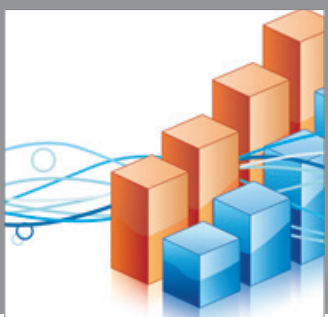

Advances in

Operations Research

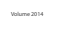

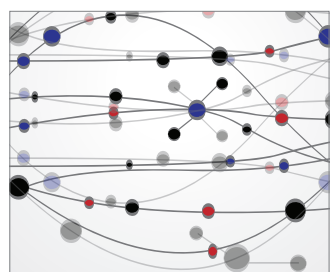

\section{The Scientific} World Journal
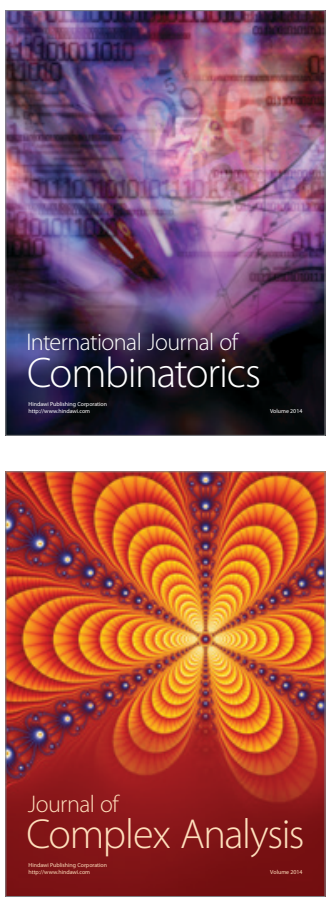

International Journal of

Mathematics and

Mathematical

Sciences
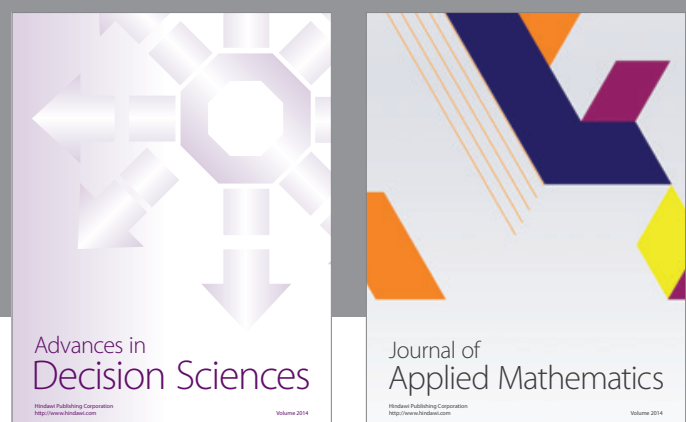

Journal of

Applied Mathematics
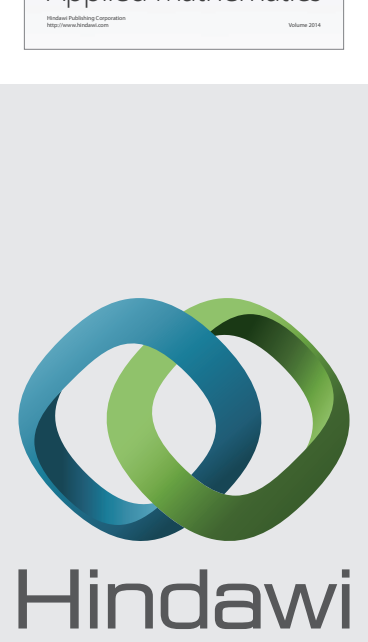

Submit your manuscripts at http://www.hindawi.com
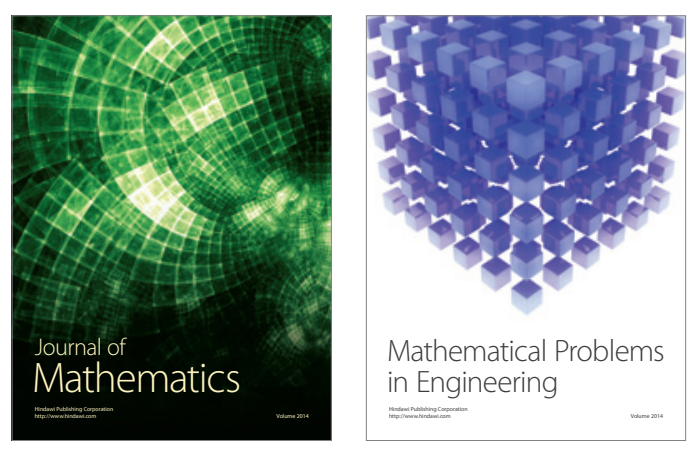

Mathematical Problems in Engineering
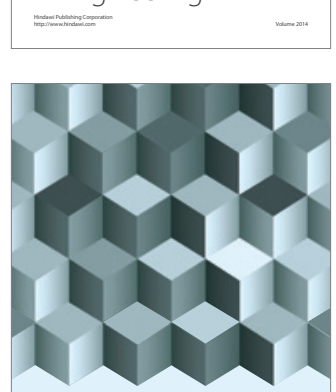

Journal of

Function Spaces
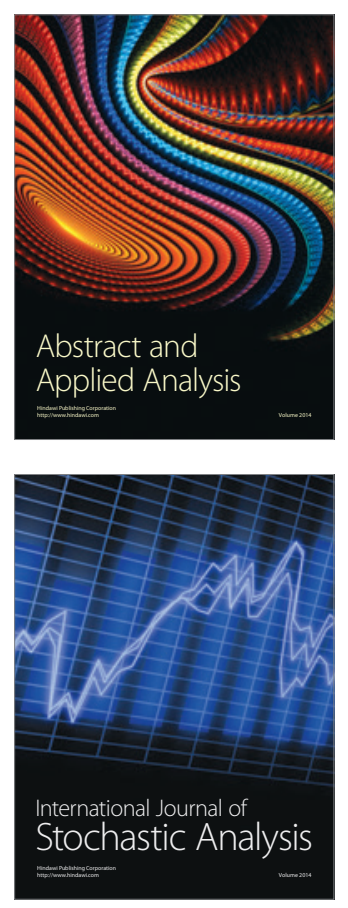

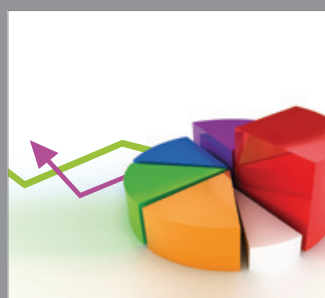

ournal of

Probability and Statistics

Promensencen
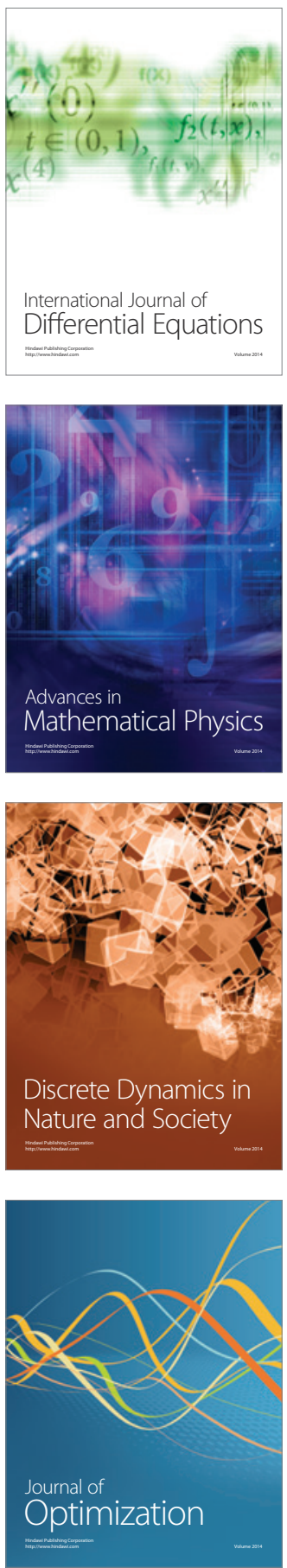